\title{
Research on Design of Intelligent Water Dispenser Based on Micro Controller System
}

\author{
Yang Yan \\ Armed Police College of CAPF, Chengdu, Sichuan, China \\ yangyan009@163.com
}

Keywords: micro controller system, intelligent water dispenser, temperature mode

\begin{abstract}
Micro controller computer is a kind of common electronic products, and it is often used for temperature detection in electronic devices. This paper will make full use of the advantage of micro controller computer to design an intelligent water dispenserwith the temperature detection and control function. In the design, we firstly determine the requirements and solutions, and then analyze the hardware configuration as well as the software program, finally debug the hardware and software in order to provide reference for relevant researchers.
\end{abstract}

\section{Introduction}

MCS (Micro Controller System) application is very broad in industry. For instance, there are intelligent electric power system based on micro controller system, intelligent vehicle speed control system based on micro controller system, intelligent data acquisition system based on micro controller system, displacement measurement system based on micro controller system and intelligent control system based on micro controller system. MCS is actually a set of RAM, CPU, ROM, input and output systems, as well as timers, counters, including micro computer system. Its micro intelligent computer system makes it possible to realize the automation and intelligence of the electric appliance. With the rise of people's attention to the health of drinking water, most families, enterprises and government units are equipped with drinking fountains. It is regrettable that the degree of intelligent water machine is not enough. The water temperature is unknown, and the level control and temperature control is not its original function.

The traditional water dispenser has two water outlets, a hot water outletwith the water temperature at 100 degrees, and the other water outlet is cold water outlet. However, under the background of people's rising living standards, it is difficult to meet the needs of people's daily life. Problems in the design of the intelligent drinking machine, aiming at cannot perceive the water to water supply induction to know the temperature, cannot adjust the temperature four current drinking machine, designed a can be perceived effluent, induction suspension of water supply, to know the temperature, regulating water temperature intelligent drinking machine. Its core components for the microcontroller based intelligent temperature control system, in order to adjust the water temperature. In the intelligent drinking machine, the user first set the required water temperature. When the water temperature reaches the setting value, the sensor can sense the water temperature, and the water temperature is transmitted to the central control chip in the form of digital signal, and the internal heating system of the drinking water machine is heated by the signal to the water temperature, until the temperature value is determined by the user. When the user is biased in favor of drinking water with high temperature, the temperature can be set higher; when the user is inclined to drinking water, the temperature can be set lower. Intelligent drinking machine can meet the requirements of users, do not need to continuously heating water or cold water to adjust the temperature. Similar to other microcontroller based instrumentation, communications equipment, ignition systems, collection devices are known as smart, the drinking machine will also be called intelligent drinking machine. If you can do more than a number of requirements, then not only will change the market structure of the drinking machine industry, but also to change the daily lives of most peopleto bring huge economic and social development. This paper will design the hardware 
configuration and software of system, and complete the debugging of system to make the water dispenser intelligent.

\section{Requirements Analysis and Solutions Determination}

We not only need the intelligent water dispenser can realize the temperature measurement and adjustment function, automatic water and water supply function, but also we should reduce the design cost and the cost of raw materials as possible as we can in order to meet the lowest cost to create the most suitable intelligent drinking machine. Thus, the core components of the above conditions for the microcontroller, we will design a microcontroller based intelligent drinking machine. For the choice of temperature sensor, we will consider the communication between single chip microcomputer and its ability to realize the digital conversion function, which reduces the design cost, raw material cost, labor cost and so on.

In terms of display circuit part, we should consider a lot of problems, such as the user's convenience, which is the primary consideration. Only to improve the user experience, to be able to sell more drinking fountains, to achieve commercial interests. Other things such as convenience of design, OLED display and LCD display are all the categories that we need to consider. In the software design, the first need to do is to achieve the system functions, the main design system control part of the main program, plus three key parts of the cycle detection, temperature detection routines and other procedures, etc.. In this process, we need to continue to carry out the software debugging, making the procedure simple and practical. Through the above analysis, we can get the solution of the prototype. The program should be a single chip microcomputer, sensor, power supply and keyboard and other components of the display circuit. We control the communication through software programming to achieve the result of controlling the total target. We can use the smart microcontroller as the core component. Here, we use the STC12C5608AD microcontroller to save money. For temperature sensor, we choose DS18B20, which has the ability of communication and data conversion, and the price is low. Display circuit part, due to drinking fountains not only need to use during the day, but is also need to use in the night. Therefore, we choose the OLED display to achieve the purpose.

\section{Design of Hardware and Software}

\section{Working Principle and Configuration of Hardware}

Firstly, we should understand the circuit of the composition in order to design it one by one. The whole circuit includes four parts: main control circuit, power supply circuit, zero crossing detection circuit and output control circuit. Of course, the main control circuit is the most important in the single chip microcomputer as the core of the circuit system, the use of software programming to achieve the default function, is the most important part of the circuit. Taking into account the microcontroller will be the water temperature of the internal water temperature detection and processing, and then through the OLED display, sensor selection as a detection subsystem; and ultrasonic module is the use of ultrasonic sensors to detect whether there is the existence of the cup and the cup and the distance, etc. The design of the water dispenser system is made of STC12C5608AD microcontroller, temperature sensor DS18B20, keyboard circuit, OLED display circuit, and output control circuit and so on.

The microcomputer system is the central nerve of the whole circuit, which is used to monitor the data acquisition process and the way of collecting the data. It is the core processor of the whole system. The main function of the single chip microcomputer is: the comparison between the detection of water temperature and water temperature difference, when the two do not match, the temperature control through the radio frequency signal, and at the same time to transmit temperature data to OLED display. Temperature sensor DS18B20 is a tool which can be used to complete the transformation of electric power to a few electric conversions. It can be used to complete the data conversion with high accuracy and anti-interference. The application prospect is very wide. The keyboard circuit is the key of communication between the user and the single chip 
computer. In the keyboard circuit, the user sets the preset water temperature, the microcontroller determines whether the signal is heated or not. Display circuit must be able to show the current water temperature and the user needs to be heated to the temperature, but also need to show the user set temperature. After meeting the above requirements, the display should also have the following functions such as showing whether there is water in the water dispense or whether the water temperature reaches the preset temperature, etc.

We use Advanced Schematic software to draw the figure of main control circuit hardware according to the above analysis:

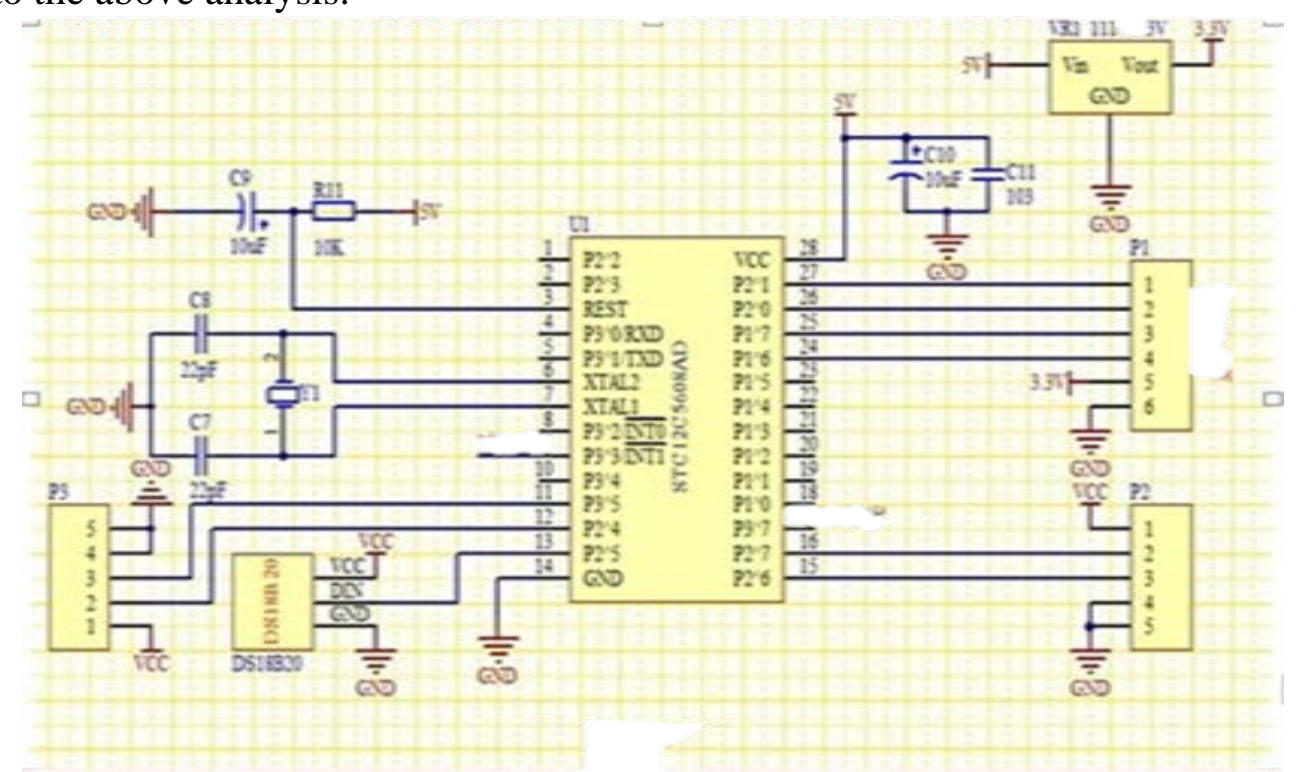

Figure 1 Figure of Main Control Circuit Hardware

\section{Design of Software Components}

The design of the software part is as important as the design of the hardware. Without the right procedure, the function of the required function cannot be achieved. Software design needs to put a lot of time and energy, because the monolithic integrated circuit is a very complex hardware system, if need to write code to carry on the control, the code is often longer. Our approach is as follows: the whole program is divided into a number of functional blocks, when these programs can be normal operation, we only need to have their organic connection can be obtained with the overall function of the module program. This method has the following three advantages: first, the single program segment relative to the whole procedure, thinking is smaller, easier to write and debug; second, when the future only needs a module function, we only need to call the module. Third, a single program is not only easy to write, and easy to debug, easy to find errors. According to the above analysis, we use the method of segmentation to design the software of the module.

\section{System Debugging of the Intelligent Water Dispenser}

\section{Hardware Debugging}

We can debug the hardware after the hardware is welded to rule out the obvious hardware failure. The first step of hardware debugging is using digital millimeter circuit for inspection is connected properly. We especially need to check the power supply part and strict inventory short circuit phenomenon. The second step is in the system power, the observation circuit is able to operate normally, the water temperature can be normal, the heating state can display, chip, pin, CPU socket and the point potential of the microcontroller. The concrete steps are as follows: the examination of power supply is very necessary, and cannot be omitted. We should test the positive and negative poles of each component by using the meter to detect the voltage of each component. In case of problems, should be immediately corrected, troubleshooting. In the power of the time, check whether the appearance of components is damaged, whether the moisture. After power, observe whether the power is connected correctly, the components and the power supply is connected, and 
so on. As for the failure of components, it should be carefully studied and debugged. Component failure may be the reason for debugging, which itself has been damaged or debugging process, due to operational errors caused by short circuit and so that components burned. Of course, the specifications of the components and the design requirements are fully in line with the installation of components is a problem we need to check the commissioning.

\section{Software Debugging}

System software debugging is an important part of system debugging. As the system is divided into modules, every module needs to be debugged. Each part of the module debugging correctly is the premise condition of the whole system debugging successfully. However, each part of the module debugging success does not mean that the overall module can run normal. Here is involved in each module of the degree of correlation, whether it can be connected closely, whether there is a logical error, etc.. In the process of debugging the software modules, the problems should be considered in the basis of the commissioning of the machine. The debugging of the module is mainly divided into zero detection part, DS18B20 program, and ultrasonic three parts. Firstly, write them into the software for running, then change, check, until you don't have a syntax error. Software debugging I use the uVision2 Kiel software, the process of compiling the process is as follows: in uVision2 Kiel, a new project, using STC series. New create a new text in uVision2 Kiel and save it masc. Add the new text to the construction project. After adding in the text to enter the written procedures and showingno error, the file can be placed in the protest simulation.

\section{Comprehensive Debugging}

Debugging the hardware and software of the system does not mean all debugging is finished. We need to debug the software and hardware at the same time to see if it can achieve the desired effect. Through the above hardware debugging, the basic rule out the system hardware problem; through the software debugging, also basic to eliminate the system software problem. In the process of comprehensive debugging of the system, we need to use the replacement method to identify the fault location, analyze the problem, and gradually eliminate the interference factor to find the reasons.

\section{References}

[1] Zhao Long, HaoRunke, Wang Lei, Qiu Yin, Hu Qian, Design of digital intelligent waterdispenser based on MCU, J., Modern Electronics Technique, 17(2012)112-115.

[2] $\mathrm{Xu}$ Ruili, PaperCupWaterLevelMeasurementor IntelligentDispenserBasedonDSP, J., Automation Application, 5(2014)1-3.

[3] Duan Qingming, Peng Xingxing, Yang Kaiqi, Lin Jie, Design of Intelligent and Safer Multi-Function TemperatureAdjustable Water Dispenser, J., Journal of Jilin University (Information Science Edition), 9(2014)16-20.

[4] XieJinxiong,ZhangDong,WeiYuying,DongBangyong, ADesignofEnergySavingDrinkingFountains BasedonMemoryControl, J., Microcomputer Information, 8(2010)25-27. 